\title{
Empleo de extracto de hibiscus sabdariffa como antioxidante natural en longaniza.
}

Use of extract of hibiscus sabdariffa as natural antioxidant in sausage.

Jairo Silva. ${ }^{1}$, Manuel Lázaro Pérez Quintana. ${ }^{2}$ \& Luis Silva. ${ }^{3}$

Recibido: 12-03-2017 / Revisado: 10-05-2017 Aceptado: 09-06-2018/ Publicado: 01-07-2018

\begin{abstract}
.
DOI: https://doi.org/10.33262/cienciadigital.v2i3.174

The use of Jamaica extract (Hibiscus sabdariffa) in different concentrations (2, 4 and $6 \%$ ) as a natural antioxidant in the production of sausage allows to offer healthy alternatives as a sausage preservative. The meat and fat, due to its composition, presents several alteration phenomena, among which the oxidation or rancidity produced by exposure to air, which causes losses in trade and industrialization, stands out. The adequate treatment of the extract of Hibiscus sabdariffa was determined by bromatological, sensory and evaluation of the antioxidant activity as a function of time. The sausage was made with pork and fat mainly as raw materials to be then ground and mixed forming a thick paste with the other ingredients. The results determined that $4 \%$ addition of Hibiscus sabdariffa extract provides acceptable bromatological and sensory characteristics. The evaluation of antioxidant activity determined that the incorporated product $4 \%$ provides better preservation qualities, a result that corroborates that of the sensory evaluation where the product presents stable appearance and acceptable coloration with the same treatment. The bromatological analysis shows variations in the concentrations of protein, fat, water, carbohydrates, minerals, when comparing the results of day 1 versus day 30 they showed that the time traveled directly influences the results, creating a reduction of each component due to dehydration in the time passed.
\end{abstract}

Keywords: Hibiscus Sabdariffa, Antioxidant Activity, Sausage, Thick Pasta, Sausage.

\section{Resumen.}

\footnotetext{
1 Universidad Estatal Amazónica, Puyo, Ecuador, vinisilva1985@hotmail.com.

2 Universidad Estatal Amazónica, Puyo, Ecuador, mpquintana1960@gmail.com.

3 Universidad Estatal Amazónica, Puyo, Ecuador, mpquintana1960@gmail.com.
} 
El empleo de extracto de Jamaica (Hibiscus sabdariffa) en diferentes concentraciones (2, 4 y 6\%) como antioxidante natural en la elaboración de longaniza permite brindar alternativas saludables como conservante del embutido. La carne y grasa por su composición presenta varios fenómenos de alteración entre los cuales destaca la oxidación o enranciamiento producido por la exposición al aire lo cual ocasiona pérdidas en el comercio e industrialización. El tratamiento adecuado del extracto de Hibiscus sabdariffa se determinó mediante pruebas bromatológicas, sensoriales y evaluación de la actividad antioxidante en función del tiempo. Se elaboró el embutido con carne de cerdo y grasa principalmente como materias primas para luego ser molidas y mezcladas formando una pasta gruesa junto a los demás ingredientes. Los resultados determinaron que el $4 \%$ de adición del extracto de Hibiscus sabdariffa proporciona aceptables características bromatológicas y sensoriales. La evaluación de actividad antioxidante determinó que el producto incorporado el $4 \%$ proporciona mejores cualidades de conservación, resultado que corrobora al de la evaluación sensorial en dónde el producto presenta apariencia estable y coloración aceptable con el mismo tratamiento. El análisis bromatológico presenta variaciones en las concentraciones de proteína, grasa, agua, carbohidratos, minerales, al ser comparados los resultados del día 1 versus día 30 demostraron que el tiempo recorrido influye directamente en los resultados creando una reducción de cada componente por la deshidratación en el tiempo transcurrido.

Palabras Claves: Hibiscus Sabdariffa, Actividad Antioxidante, Embutido, Pasta Gruesa, Longaniza.

\section{Introducción.}

La carne es un alimento importante para la dieta humana, su gran riqueza nutritiva está formada por el alto contenido en proteínas de elevado valor biológico, pero, por otro lado, es uno de los alimentos más perecederos debido a su alto contenido en agua, composición y $\mathrm{pH}$, lo que favorece la alteración y contaminación microbiana, pudiendo constituir un riesgo para la salud (Tiburcio, 2018).

La carne por su valor nutritivo y composición es un alimento altamente perecible, entre los problemas habituales se encuentra la oxidación del tejido adiposo y muscular, fenómeno que altera la composición bioquímica de la carne y presenta cambios de color reduciendo su importancia industrial. Pérez (2000) señala que el color es el factor que más afecta el aspecto de la carne y los productos cárnicos durante su almacenamiento y el que más influye en la preferencia del cliente, por lo que la alteración del color bien puede ser la causa más importante que define la durabilidad de los productos cárnicos.

Las industrias han empleado diversas técnicas para prevenir la oxidación de las carnes, aditivos como los antioxidantes industriales y naturales juegan un rol importante en la 
industria cárnica. Valenzuela (2016) menciona que los antioxidantes naturales son sustancias que se adicionan a los alimentos de origen cárnico para evitar el "enranciamiento", problema que origina decoloración, sabor desagradable y produce elementos nocivos para la salud. El sistema de defensa antioxidante está constituido por un grupo de sustancias que al estar presente en concentraciones bajas con respecto al sustrato oxidable, retrasan o previenen significativamente la oxidación de este (Pace, 2010).

La planta de jamaica (Hibiscus sabdariffa) contiene diversos compuestos. Entre los que se hallan alcaloides, ácido ascórbico, anisaldehído, antocianinas, b-caroteno, ácido cítrico, ácido málico, galactosa, pectina, ácido esteárico (Hirunpanich et al. 2005). A los extractos que se obtienen a partir de éstos se les han atribuido diversas propiedades medicionales como efectos diuréticos, coleréticos, reducción de la presión arterial, reducción de los niveles de colesterol (Duke et al. 2003). Por la actividad antioxidantes de sus antocianinas, también pueden ayudar en la prevención y tratamiento de algunos tipos de cáncer (Chen et al. 2003).

Con la información descrita se formuló longaniza adicionando extracto de Hibiscus sabdariffa (2, 4 y 6\%) para evaluar su actividad antioxidante, se analizó el porcentaje adecuado que brinda el mayor tiempo de conservación en percha, se evaluó la calidad mediante pruebas bromatológicas, sensoriales y la actividad antioxidante en función del tiempo.

\section{Materiales y métodos.}

\section{Localización.}

La presente investigación tuvo efecto en el laboratorio de Agroindustrias, Carrera de Agroindustria de la Universidad Estatal Amazónica. Localizado en el Km. 2. 1/2 vía Puyo a Tena (Paso Lateral). Provincia de Pastaza entre las coordenadas $0^{\circ} 59^{\prime}-1$ ' S y a una longitud de $77^{\circ} 49^{\prime}$ ' ' W, se encuentra en la Región Amazónica del Ecuador, en el occidente de la provincia de Pastaza a unos 924 m.s.n.m. Temperatura 18 a 24 ${ }^{\circ} \mathrm{C}$.

\section{Obtención de extractos de antocianinas (antioxidante natural).}

Agua destilada. Se colocan $10 \mathrm{~g}$ con base $12 \%$ de humedad de la muestra en un vaso de precipitado de $500 \mathrm{~mL}$ y se agregaron $100 \mathrm{~mL}$ de agua destilada. Los vasos se colocaron en una parrilla con un sistema de enfriamiento acoplado sobre la boca del vaso para evitar la pérdida de agua por evaporación y se dejaron ebullir por 16 y 60 min, considerando como to al tiempo en que la mezcla alcanzó la ebullición plena (Galicia et al. 2008). 
Metanol acidificado. Las antocianinas se extrajeron a partir de $1 \mathrm{~g}$ de muestra molida a la que se añadieron $20 \mathrm{~mL}$ de metanol acidificado al $1 \%$ con ácido trifluoracético y se mantuvo durante 24 horas bajo condiciones de refrigeración. Posteriormente se realizaron dos extracciones adicionales empelando como disolvente metanol:ácido acético:agua, (10:1:9 v/v), manteniendo bajo agitación y a temperatura durante 24 horas.

\section{Elaboración de la longaniza fresca.}

La longaniza fresca fue elaborada según lo indicado en la tabla 1 con la adición del extracto de Hibiscus sabdariffa a diferentes concentraciones. Fórmula sugerida en la investigación realizada por Silva et al. (2018) sobre polifenoles de Theobroma cacao L. como antioxidante natural en chorizo fresco.

Tabla 1. Formulación de longaniza con extracto de Hibiscus sabdariffa.

\begin{tabular}{lcccc}
\hline & \multicolumn{4}{c}{ ANTIOXIDANTE $(\%)$} \\
\cline { 2 - 5 } \multicolumn{1}{c}{ INGREDIENTES } & 0,00 & 2,00 & 4,00 & 6,00 \\
\hline Carne Cerdo & 55,00 & 55,00 & 55,00 & 55,00 \\
Grasa & 35,00 & 35,00 & 35,00 & 35,00 \\
Hielo/agua & 9,00 & 9,00 & 9,00 & 9,00 \\
Almidón & 1,00 & 1,00 & 1,00 & 1,00 \\
\hline TOTAL M.P. & 100,00 & 100,00 & 100,00 & 100,00 \\
\hline & & & & \\
Sal & 2,20 & 2,20 & 2,20 & 2,20 \\
2Sal cura & 0,33 & 0,33 & 0,33 & 0,33 \\
Fosfato & 0,25 & 0,25 & 0,25 & 0,25 \\
Antiox. & 0,00 & 0,02 & 0,04 & 0,06 \\
Cond. & 0,95 & 0,95 & 0,95 & 0,95 \\
Pimienta negra & 0,30 & 0,30 & 0,30 & 0,30 \\
Ajo & 0,10 & 0,10 & 0,10 & 0,10 \\
Color & 0,04 & 0,04 & 0,04 & 0,04 \\
\hline TOTAL COND. & 4,17 & 4,19 & 4,21 & 4,23 \\
\hline
\end{tabular}

Elaborado por: Grupo de investigación.

En el estudio se tomaron muestras de cada tratamiento en el día 1 y después de 30 días, se analizó y comparó resultados de las evaluaciones sensoriales, bromatológicas y actividad antioxidante. 


\section{Resultados y discusión.}

Análisis sensorial. El análisis sensorial se realizó con 20 degustadores que determinaron el grado de aceptabilidad de las longanizas como se muestran en la tabla 2. Los tratamientos fueron codificados y ubicados alternadamente.

Tabla 2. Análisis sensorial de longaniza con extracto de Hibiscus sabdariffa.

\begin{tabular}{|c|c|c|c|c|c|c|c|c|}
\hline \multirow{4}{*}{$\begin{array}{c}\text { Nivel de } \\
\text { agrado }\end{array}$} & \multicolumn{8}{|c|}{ Longaniza con extracto de Hibiscus sabdariffa. } \\
\hline & \multicolumn{4}{|c|}{ DÍA 1} & \multicolumn{4}{|c|}{ DÍA 30} \\
\hline & 0 & 2 & 4 & 6 & 0 & 2 & 4 & 6 \\
\hline & $\%$ & $\%$ & $\%$ & $\%$ & $\%$ & $\%$ & $\%$ & $\%$ \\
\hline $\begin{array}{l}\text { Me gusta } \\
\text { mucho }\end{array}$ & 6 & 7 & 9 & 8 & 2 & 6 & 7 & 5 \\
\hline Me gusta & 7 & 7 & $\begin{array}{l}1 \\
0\end{array}$ & 7 & 4 & 4 & 7 & 6 \\
\hline $\begin{array}{lr}\text { Ni me } \\
\text { gusta ni } \\
\text { me } \\
\text { disgusta }\end{array}$ & 5 & 5 & 2 & 3 & 4 & 5 & 4 & 8 \\
\hline $\begin{array}{l}\text { No me } \\
\text { gusta }\end{array}$ & 2 & 1 & 0 & 2 & 8 & 3 & 2 & 1 \\
\hline $\begin{array}{l}\text { Me } \\
\text { disgusta } \\
\text { mucho }\end{array}$ & 0 & 0 & 0 & 0 & 2 & 2 & 0 & 0 \\
\hline \multirow{2}{*}{ TOTAL } & 2 & 2 & 2 & 2 & 2 & 2 & 2 & 2 \\
\hline & 0 & 0 & 0 & 0 & 0 & 0 & 0 & 0 \\
\hline
\end{tabular}

Elaborado por: Grupo de investigación.

Los análisis sensoriales demuestran que la longaniza con extracto de Hibiscus sabdariffa en el día 1 presentó mayor acogida con el 4\% de adición del antioxidante natural; en el día 30 la longaniza con el tratamiento del $4 \%$ también presenta resultados favorables.

Actividad antioxidante. El método para determinar la actividad antioxidante fue la estabilización del 2,2 - difenil - 1 - picrilhidrazil (DPPH) que se basa en la medición de la habilidad de compuestos antioxidantes para reducir el DPPH a partir del decremento en absorbancia de la reacción, en función del tiempo (Prior et al. 2005). 
Los resultados de la actividad antioxidante se presentan en la tabla 3. Los incrementos de los valores de actividad antioxidante se debe a la actividad antioxidante de los compuestos fenólicos y sus propiedades redox, las cuales desempeñan un papel importante en la adsorción y neutralización de radicales libres que aparecen con la presencia de oxígeno y a medida que transcurre el tiempo (Murthy et al. 1998).

Tabla 3. Actividad antioxidante en longaniza con extractos de Hibiscus sabdariffa $(\mathrm{mg} / \mathrm{kg})$.

\begin{tabular}{ccc}
\hline Longaniza & Día 1 & Día 30 \\
\hline $0 \%$ & $0.000^{\mathrm{a}}$ & $0.000^{\mathrm{c}}$ \\
$2 \%$ & $0.200^{\mathrm{a}}$ & $0.280^{\mathrm{a}}$ \\
$4 \%$ & $0.223^{\mathrm{a}}$ & $0.358^{\mathrm{b}}$ \\
$6 \%$ & $0.239^{\mathrm{a}}$ & $0.365^{\mathrm{b}}$ \\
\hline
\end{tabular}

Elaborado por: Grupo de investigación.

${ }^{\mathbf{a}, \mathbf{b}}$ Promedios con letras diferentes difieren estadísticamente (Tukey $\mathrm{p}<0.05$ ).

La relación de la actividad antioxidante obtenidas en este trabajo son similares a los obtenidos por Silva et al. (2018) en su estudio sobre polifenoles de la almendra de Theobroma cacao L. como antioxidante natural en chorizo fresco, dónde a partir de la actividad inicial con el transcurso del tiempo existe un incremento relativo de 0.216 a 0.388 $(\mathrm{mg} / \mathrm{kg})$, de 0.219 a $0.413(\mathrm{mg} / \mathrm{kg}), 0.251$ a 0.410 , valores obtenidos con el 2,4 у $6 \%$ respectivamente de incorporación del antioxidante natural.

\section{Análisis bromatológico.}

El análisis bromatológico se realizó para evaluar el contenido físico - químico del producto, variables como proteína, grasa, agua y minerales son importantes cuando de información nutricional se trata. Los resultados obtenidos se muestran en la tabla 4. 
Tabla 4. Análisis bromatológico de longaniza con extractos de Hibiscus sabdariffa.

\begin{tabular}{|c|c|c|c|c|c|}
\hline \multirow[b]{2}{*}{ VARIABLE } & $\mathrm{T}$ & \multicolumn{4}{|c|}{ TRATAMIENTOS } \\
\hline & $\begin{array}{l}\mathrm{M} \\
\mathrm{P} \\
\mathrm{O}\end{array}$ & $\begin{array}{l}0 \\
\%\end{array}$ & $\begin{array}{l}2 \\
\%\end{array}$ & $\begin{array}{l}4 \\
\%\end{array}$ & $\begin{array}{l}6 \\
\%\end{array}$ \\
\hline \multirow{8}{*}{$\begin{array}{l}\text { Proteína bruta } \\
\text { g/100g }\end{array}$} & Día 1 & 2 & 2 & 2 & 2 \\
\hline & & 0 & 2 & 3 & 2 \\
\hline & & , & 5 & 8 & , \\
\hline & & 4 & 3 & 8 & 6 \\
\hline & Día & 1 & 2 & 2 & 2 \\
\hline & 30 & 9 & 2 & 2 & 2 \\
\hline & & 9 & 3 & 5 & 7 \\
\hline & & 8 & 3 & 6 & 5 \\
\hline \multirow{10}{*}{$\begin{array}{l}\text { Grasa total } \\
\mathrm{g} / 100 \mathrm{~g}\end{array}$} & Día 1 & 2 & 2 & 2 & 2 \\
\hline & & 9 & 7 & 6 & 8 \\
\hline & & , & , & , & , \\
\hline & & 2 & 6 & 3 & 0 \\
\hline & & 7 & 6 & 3 & 5 \\
\hline & Día & 2 & 2 & 2 & 2 \\
\hline & 30 & 7 & 5 & 4 & 5 \\
\hline & & , & , & , & , \\
\hline & & 6 & 0 & 0 & 6 \\
\hline & & 3 & 0 & 2 & 0 \\
\hline \multirow{10}{*}{ Agua g/100g } & Día 1 & 4 & 3 & 3 & 3 \\
\hline & & 0 & 9 & 9 & 7 \\
\hline & & , & , & , & , \\
\hline & & 7 & 4 & 2 & 0 \\
\hline & & 6 & 4 & 4 & 8 \\
\hline & Día & 3 & 3 & 3 & 3 \\
\hline & 30 & 6 & 5 & 3 & 2 \\
\hline & & , & , & , & , \\
\hline & & 2 & 5 & 5 & 2 \\
\hline & & 5 & 6 & 6 & 2 \\
\hline \multirow{4}{*}{$\begin{array}{l}\text { Minerales } \\
\mathrm{g} / 100 \mathrm{~g}\end{array}$} & Día 1 & 4 & 4 & 4 & 4 \\
\hline & & , & , & , & , \\
\hline & & 5 & 7 & 2 & 7 \\
\hline & & 5 & 3 & 4 & 5 \\
\hline
\end{tabular}


Carbohidratos

$\mathrm{g} / 100 \mathrm{~g}$
Día

30

Día 1

30

\section{4}

,

4

4

$\begin{array}{lll}4 & 4 & 4 \\ 3 & , & 3 \\ 3 & 5 & 3 \\ 2 & 6 & 3\end{array}$

$\begin{array}{llll}9 & 1 & 1 & 1\end{array}$

$\begin{array}{llll}0 & 3 & 1\end{array}$

$\begin{array}{llll}0 & 1 & 5 & 5\end{array}$

5

5

30

$\begin{array}{llll}8 & 9 & 1 & 1 \\ , & , & 2 & 1 \\ 5 & 3 & 5 & 2 \\ 4 & 4 & 5 & 2 \\ \end{array}$

Elaborado por: Grupo de investigación.

La FAO (2017) señala que el chorizo y longaniza son embutidos crudos, de textura heterogénea y de origen español elaborados a partir de carne picada de cerdo revuelta con sal, especias y nitrato de potasio. Al ser un producto de pasta gruesa es difícil obtener resultados semejantes del mismo producto y existen variaciones como se muestra en la figura 1 .

Figura 1. Contenido de proteína en longaniza con extractos de Hibiscus sabdariffa.

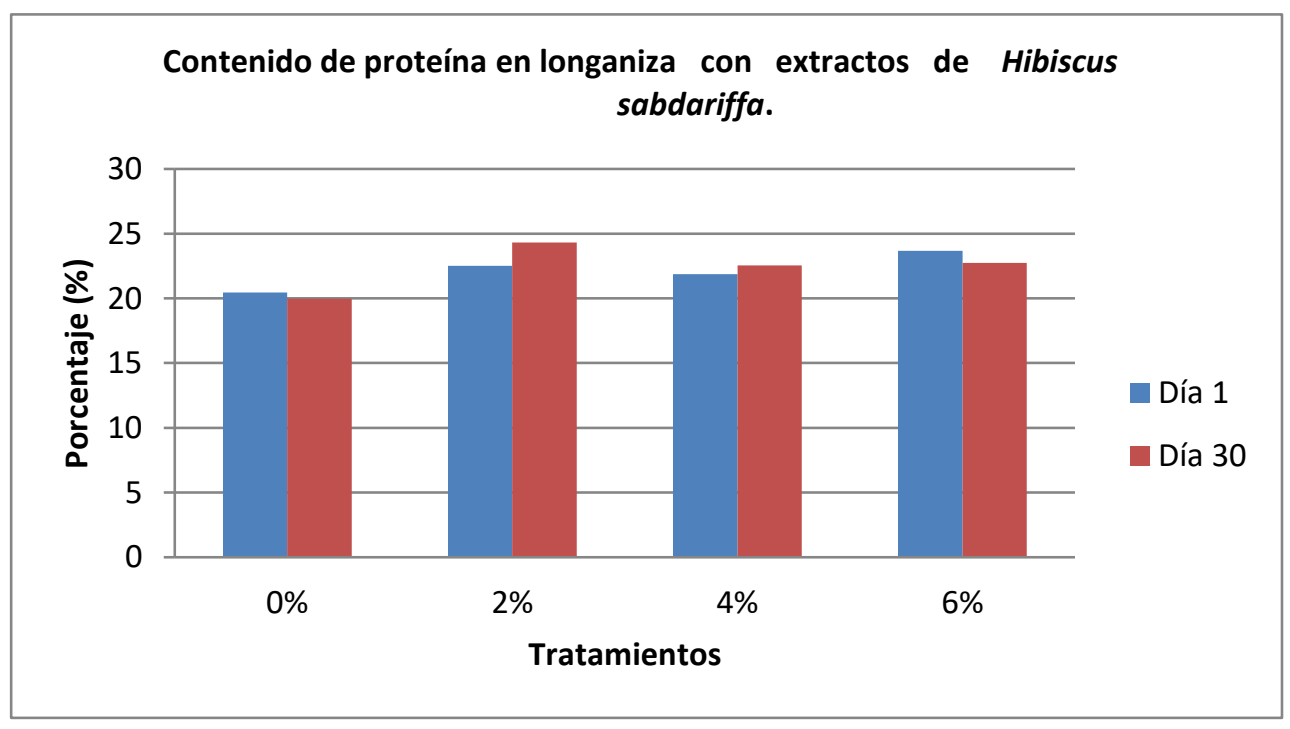

Elaborado por: Grupo de investigación. 


\section{Conclusiones.}

- La longaniza elaborada con el 6\% del extracto de Hibiscus sabdariffa presenta un existe un incremento de la actividad polifenólica el cual indica su presencia y por ende actividad antioxidante.

- En el análisis bromatológico, la evaluación de proteína (g/100g) presenta una mayor concentración es el tratamiento con el $4 \%$ del extracto incorporados, mientras que con el $2 \%$ no existe un mayor cambio al igual que el $6 \%$.

- La evaluación sensorial determinó que el $4 \%$ es el tratamiento que mejores características organolépticas brinda el producto a los consumidores; el 6\% también proporciona cualidades especiales y finalmente el $2 \%$ no presenta mayor cambio en el producto.

- El empleo del extracto de Hibiscus sabdariffa como antioxidante natural incorporado en fórmulas con el $4 \%$ brinda propiedades de conservación estables, dicho porcentaje puede ser empleado en la elaboración de varios productos de origen cárnico.

\section{Referencias bibliográficas.}

Chen, C. Wang, S. Chiang, H. (2003). Hibiscus sabdariffa Extract inhibits the development of atherosclerosis in cholesterol-fed rabbits. Journal og Agriculture and Food Chemistry. 51, 5472-5477.

Duke, J. Ducellier, J. (2003). Handbook of medicinal spices. CRC Press LLC. New York, USA. 348 p.

Galicia, L. Salinas, Y. Espinoza, B. Sánchez, C. (2008). Caracterización fisicoquímica y actividad antioxidante de extractos de Jamaica (Hibiscus sabdariffa L.) nacional e importada. Revista Chapingo Serie Horticultura 14(2): 121 - 129.

Hirunpanich, V. Morales, N. Sato, H. Suthinsisang, C. (2005). Antioxidant effect of aqueous extracts from dried calyx of Hibiscus sabdariffa Linn. (Roselle) in vitro using rat low-density lipoprotein (LDL). Biological and Pharmaceutical Bulletin. 28(3). 481-484.

Murthy, B. Murch, S. Saxena, P. (1998). Thidiazuron: a potent regulator of in vitro plant morphogenis. In Vitro Cell Dev Biol Plant.34 (6):267-75.

Organización de las Naciones Unidas para la Alimentación y la Agricultura (FAO). (2017). Fichas Técnicas. Procesados de carne. 
Pace, V. (2010). Universidad abierta interamericana. Antioxidantes naturales. p39.

Pérez, D. Andújar, G. (2000). Instituto de Investigaciones para la Industria Alimenticia. Cambios de coloración de los productos cárnicos. Rev. Cubana Aliment Nutr 2000;14(2):114-23.

Prior, R. We, X. Schiaich, K. (2005). Standarized methodos for the determination of antioxidant capacity and phenolics in foods and dietary supplements. Jourdal of Agriculture and Food Chemistry. 53, 4290-4302.

Silva, L. Lázaro, M. \& Bravo, L. (2018). Polifenoles de la almendra de theobroma cacao L. como antioxidante natural en chorizo fresco. Revista electrónica Ciencia Digital 2(2), 424-437.

Tiburcio, C. (2018). Zona económica. Características de la Carne como Alimento. Recuperado https://www.zonaeconomica.com/carne-alimento.

Valenzuela, V. Carolina, C. y Pérez, M. (2016). Actualización en el uso de antioxidantes naturales derivados de frutas y verduras para prolongar la vida útil de la carne y productos cárneos. Rev. chil. nutr. [online]. 43,(2): 188-195.

\section{Ciencia
Digital
$\varepsilon_{\text {edtroul }}$}




\section{Para citar el artículo indexado.}

Silva J., Pérez M. \& Silva L. . (2018). Empleo de extracto de hibiscus sabdariffa como antioxidante natural en longaniza. Revista electrónica Ciencia Digital 2(3), 329-339. Recuperado http://cienciadigital.org/revistacienciadigital2/index.php/CienciaDigital/article/view/174/15 $\underline{4}$

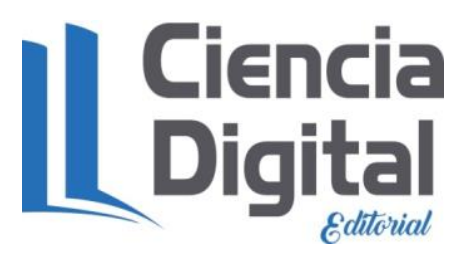

El artículo que se publica es de exclusiva responsabilidad de los autores y no necesariamente reflejan el pensamiento de la Revista Ciencia Digital.

El articulo queda en propiedad de la revista y, por tanto, su publicación parcial y/o total en otro medio tiene que ser autorizado por el director de la Revista Ciencia Digital.
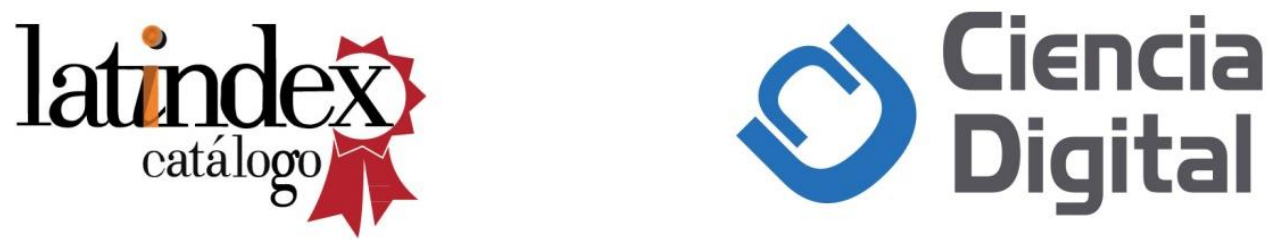